\title{
Clinical evaluation of scanning laser polarimetry: II Polar profile shape analysis
}

\author{
Andrew Waldock, Michael J Potts, John M Sparrow, Wojciech S S Karwatowski
}

\begin{abstract}
Aims-To devise a method to describe and quantify the shape of polar profiles obtained with the scanning laser polarimeter and to compare this measurement with other polar profile measurements in a series of normal subjects and glaucoma patients.
\end{abstract}

Methods-Scanning laser polarimetry was performed on 54 normal subjects and 74 glaucoma patients. The retardation values obtained from one randomly chosen eye of each subject were analysed using our own methods, including the use of an algorithm to remove blood vessels from the polar profiles, an algorithm to standardise the glaucoma profiles to a normal database, and a further algorithm to evaluate the profile shape. The measurements of profile shape were compared with measurements of the absolute and standardised retinal nerve fibre layer thickness obtained with the scanning laser polarimeter.

Results-There was no significant difference between the mean retardation values for the normal and glaucomatous subjects in either hemiretina. However, standardisation of the glaucoma retardation values to a normal database produced significant differences at $p<1 \times 10^{-8}$ in the mean retardation values for these two groups in both hemiretinas. Profile shape measurement analysis produced similar significant differences between the mean retardation values for the normal and glaucomatous subjects in both hemiretinas, although the degree of separation was greater following standardisation of the retardation values.

Conclusion-The use of an algorithm to standardise an individual's retardation values in conjunction with a blood vessel removal algorithm enables an improvement in the ability of the scanning laser polarimeter to discriminate between normal and glaucomatous patients. The polar profile shape algorithm is independent of standardisation and significantly improves the discrimination between normal and glaucomatous patients, as well as providing additional information regarding the retinal nerve fibre layer.

(Br f Ophthalmol 1998;82:260-266)

Infirmary, Leicester

W S S Karwatowski

Correspondence to:

Mr Andrew Waldock, Torbay

Hospital, Lawes Bridge,

Torquay, Devon, TQ2 7AA.

Accepted for publication 10 October 1997

The scanning laser polarimeter (Nerve Fiber Analyzer, Laser Diagnostic Technologies, San Diego, CA, USA) is one of the new computerised image analysing techniques ${ }^{12}$ which have been developed in an attempt to provide a more reliable, objective, and quantitative measurement of the retinal nerve fibre layer. It utilises the properties of polarised light to measure the thickness of the birefringent retinal nerve fibre layer. ${ }^{3}$ The underlying principle assumes that the birefringence of the retinal nerve fibre layer causes a change in the state of the polarised light (known as retardation). This retardation is measured by the instrument and converted into a measure of the retinal nerve fibre layer thickness based upon the assumption that there is a linear relation between the thickness and optical properties of this layer. ${ }^{3}$

Early studies have assessed the retardation values obtained with the scanning laser polarimeter from a population of normal and glaucomatous subjects, ${ }^{4}$ as well as comparing retardation values with visual field loss ${ }^{5}$ and with standardised red-free photography. ${ }^{6}$ Initial research performed by the authors has attempted to improve the interpretation of the raw data obtained with the scanning laser polarimeter. ${ }^{7-10}$ This has involved the design and evaluation of a blood vessel removal algorithm ${ }^{78}$ and an evaluation of the pattern and distribution of the retardation values obtained with this technology ${ }^{910}$ in a cohort of normal and glaucoma patients.

One of the most useful variables describing the retinal nerve fibre layer appears to be an assessment of the shape of this anatomical structure. ${ }^{9}{ }^{10}$ The initial research demonstrated that there was relatively poor discrimination between the "raw" retardation values obtained from the normal and glaucomatous subjects. ${ }^{9}{ }^{10}$ However, it also highlighted the potential that "standardising" the data to defined regions and assessing the "polar profile shape" have in discriminating between normal and glaucomatous eyes. ${ }^{910}$ In this study, a simple algorithm has been used to standardise an individual's profile to a normal database. Furthermore, a more sophisticated algorithm has been devised to describe the shape of the polar profiles obtained with the scanning laser polarimeter. These algorithms have been applied to the profiles of a group of normal and glaucomatous eyes and their performance has been compared with the more conventional analysis of the polar profiles.

\section{Patients and methods}

PATIENTS
Fifty four normal subjects (20 male, 34 female) with healthy eyes and an age range of 31 to 89 years were examined. These were compared with 74 patients (49 male, 25 female) with 
primary open angle glaucoma recruited from the glaucoma clinics at Bristol Eye Hospital. These had an age range from 49 to 83 years. The mean ages for the two groups was 63 years (normal) and 70 years (glaucoma) respectively. This cross sectional study was approved by the ethics committee for the United Bristol Healthcare Trust and informed consent was obtained from all of the patients.

Normal subjects were included in the study if there was no family history of glaucoma and they had had no intraocular pressure rise or ocular trauma in the past, a best corrected visual acuity of $6 / 12$ or better, normal anterior segments on slit lamp biomicroscopy, intraocular pressure of less than $22 \mathrm{~mm} \mathrm{Hg}$ (Goldmann tonometer), normal optic nerve head appearances on stereoscopic optic disc photography, and no glaucomatous visual field defects on Humphrey 24-2 full threshold automated perimetry.

Primary open angle glaucoma patients were included if they had a best corrected visual acuity of $6 / 12$ or better, anterior segment examination which confirmed open angles but did not demonstrate pseudoexfoliation or pigment dispersion, had glaucomatous changes to the appearance of their optic nerve heads on stereoscopic optic disc photography, and had visual field abnormalities consistent with glaucoma. These visual field defects were defined by at least three adjacent points $5 \mathrm{~dB}$ or more below the normal with a Humphrey 24-2 full threshold program.

SCANNING LASER POLARIMETER

Retardation was measured with a Mark I scanning laser polarimeter (Nerve Fiber Analyzer (NFA I)). ${ }^{11}$ The technical details regarding this instrument have been documented previously ${ }^{23}$ and have been described in detail elsewhere. ${ }^{8}$ In essence, a near infrared diode laser (wavelength of $780 \mathrm{~nm}$ ) is used as the light source for a scanning laser ophthalmoscope with a polarisation modulator, corneal polarisation compensator, and polarisation detection unit integrated into the system. The modulated polarised laser beam is focused onto the retina and undergoes refraction by the birefringent retinal nerve fibre layer, reflection by the deeper retinal layers, and further refraction before emerging from the eye in a different state of polarisation. This emergent beam is analysed by a polarisation detection unit and the output stored in an accompanying microprocessor.

In this study a 15 degree field of view was used to illuminate the retina. An image consisting of $256 \times 256$ pixels and containing retardation data for these 65536 retinal positions is acquired in less than 1 second. The microprocessor uses an algorithm to convert the retrieved retardation data from each measured position into a retardation map (image) which displays the state of polarisation at each location within the field of view. This takes approximately 15 seconds and can be displayed in a variety of pseudocolour coded scales.
The protocol for retrieving retardation values from the image has yet to be standardised by the manufacturers. In this study, the optic disc margin of each eye was defined by placing a circle around the inner margin of the peripapillary scleral ring by a single experienced operator. Retardation values were subsequently obtained for six circles of 1.5 to 2.0 disc diameters in dimension. For each circle, the "band width" function was set at 1 and the "sector" function set to 360. In essence, this means that for each of the degrees comprising a circle of data, only one retardation value was used for each angular degree.

The retardation values obtained by the above method were transferred from the integrated microprocessor to a personal computer for further calculation. A polar coordinate plot (polar profile) was calculated for each eye by obtaining a mean retardation value for each of the 360 degrees. This mean value derives from the average of the retardation values obtained at each degree for the six circles.

\section{CLINICAL STUDY}

In this study, optic disc cupping was graded by two independent observers using stereoscopic optic disc photographs. A template of diagrams depicting the full range of cup to disc ratios was used as a reference by both of the observers. The mean of the vertical and horizontal components of the cup:disc ratio from both observers was taken to represent the cup:disc ratio. All subjects had three "good quality" images obtained from each eye by one experienced operator in a single session using the scanning laser polarimeter. The criteria for "good quality" images included the absence of any eye movement during the image acquisition, the presence of "even illumination" of the whole image, and optimal use of the dynamic range of the polar profiles. In all imaging sessions, the patients rested after each image acquisition and subsequently repositioned themselves for each further image. A complete image acquisition series of both eyes and three images per eye would take between 10 and 15 minutes.

The polar profiles obtained from one randomly chosen eye from each of the subjects were analysed in the following way. The three polar profiles from the chosen eye of each of the subjects were initially aligned using the author's own "best fit" algorithm based upon a "least squares" method. In essence, this algorithm comprises the calculation of the sum (designated "s") of the square of the differences in retardation values at each of the 360 points comprising a polar profile for a specified alignment of the three polar profiles. The alignment of the three profiles is then modified in a systematic fashion and the process repeated. The alignment with the lowest "s" value is defined as having the best fit. A mean polar profile was subsequently created from these three aligned profiles.

The presence of blood vessels within these profiles has been demonstrated to be a source of high measurement variability by the authors $^{78}$ and also a feature which inhibits the 

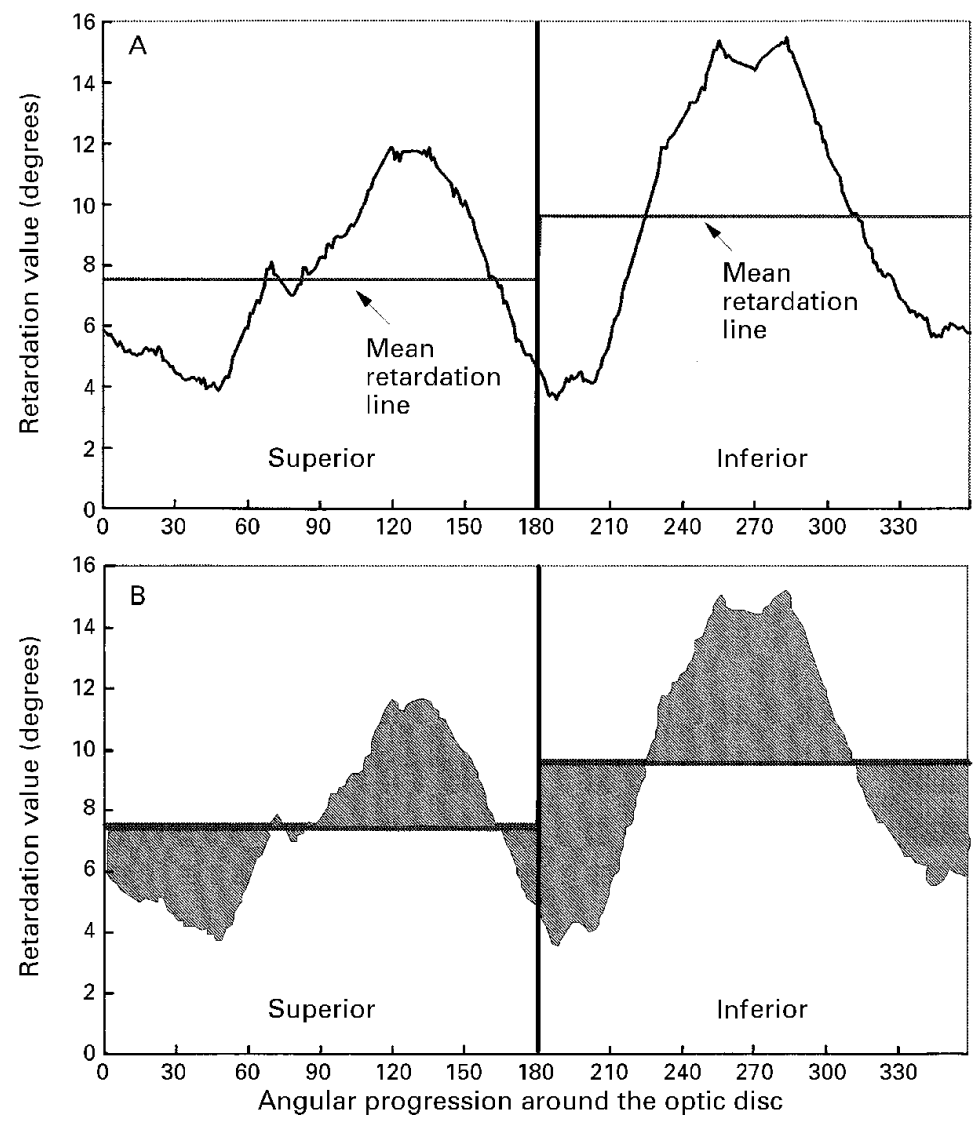

Figure 1 (A) Example of a polar profile of a normal subject demonstrating the mean retardation value for each hemiretina. (B) Example of the same normal polar profile demonstrating the application of the algorithm to calculate the profile shape (depicted as the shaded area of the profile). $0=$ Temporal midline; $90=$ superior midline; $180=$ nasal midline; 270 = inferior midline.

description of the polar profile shape. The authors have devised an algorithm which removes the blood vessels from the polar profiles without oversmoothing the rest of the profile. The full details of this algorithm and its performance have been described elsewhere. ${ }^{78}$ In brief, the algorithm is designed to detect blood vessels within the profile by assessing gradient changes within the profile. If the threshold for a gradient change is exceeded, the algorithm recognises this as the presence of a blood vessel. The algorithm extrapolates across the region defined by the above process as the site of a blood vessel. This process is performed on several occasions, initially assessing the profile in a clockwise (1-360 degrees) and then an anticlockwise direction (360-1 degree). The presence of vessel bifurcations and trifurcations is also recognised and this requires further processing of the profiles by this algorithm using different gradient thresholds depending upon the nature of the vessel composition.

Table 1 Demographic data for the eyes analysed in the study

\begin{tabular}{lllllll}
\hline & No & $M / F$ & $\begin{array}{l}\text { Age } \\
\text { (years) }\end{array}$ & Eyes $R / L$ & Cup:disc ratio & $\begin{array}{l}\text { Visual field } \\
(M D)\end{array}$ \\
\hline Normal & 54 & $20 / 34$ & 63 & $28 / 26$ & 0.55 & $0 \mathrm{~dB}$ \\
Glaucoma & 74 & $49 / 25$ & 70 & $37 / 37$ & 0.75 & $-8.25 \mathrm{~dB}$ \\
\hline
\end{tabular}

Age, cup:disc ratio, and visual field documented as mean values for the normal and glaucomatous groups.
The mean aligned polar profiles of all subjects underwent the removal of blood vessels using the aforementioned algorithm. A further realignment was performed to enable direct comparison of every profile with regard to the nasal minimum. This is calculated by finding the lowest value of an average of 20 degrees based around a midpoint ranging from 20 degrees above the horizontal nasal midline to 20 degrees below the horizontal nasal midline. The final location of the nasal minimum in relation to the horizontal midline is registered for further reference, while the polar profiles are realigned around this nasal minimum.

Previous research by the authors ${ }^{9}{ }^{10}$ has demonstrated that this instrument appears to measure "relative" retinal nerve fibre layer thickness. It was observed that in patients with increasing glaucomatous nerve fibre loss, the superior and inferior polar profile peaks were less prominent while the nasal and temporal troughs appeared to be less deep. ${ }^{9}{ }^{10}$ This unusual phenomenon could be overcome by "standardising" the superior and inferior retardation measurements to the nasal quadrant. ${ }^{9}{ }^{10}$

In this study it was decided that the effect of "standardising" an individual's polar profile should be formally assessed. The normal subjects' polar profiles were separated into right and left eyes and a mean polar profile from all the subjects was created for each eye. This created an eye matched normal database. The retardation values from the nasal 30 degrees and temporal 30 degrees were then averaged to obtain a "reference" retardation value from which each of the individual glaucomatous eyes could be standardised.

The process of standardisation requires the alignment of an individual's profile to the normal database profile, using the nasal minimum as the reference point. Once this has been performed, the mean retardation value from the individual's nasal 30 degrees and temporal 30 degrees is compared with the normal reference value and a ratio obtained. This ratio is then applied to each of the 360 retardation values comprising the individual's polar profile.

In an earlier study, ${ }^{910}$ the authors devised relatively crude methods for describing the profile shape. In this study, the profile shape has been calculated by using the mean retardation values for the hemiretinas (as described previously), and subsequently calculating the absolute difference between this value and each of the 180 retardation values comprising a hemiretina. The profile shape measurement for each hemiretina is then expressed as the sum of these absolute differences from the mean. An example of this simple algorithm is illustrated in Figure 1.

This relatively complex series of analyses of the raw retardation values from normal and glaucomatous eyes enables the data to be expressed and evaluated in several ways. In essence, the retardation values contained within the blood vessel removed and realigned polar profiles can be expressed in terms of a mean retardation value for the superior and inferior hemiretinas. These values represent a 
Table 2 Retardation values and profile shape values

\begin{tabular}{llllll}
\hline & Normal & $\begin{array}{l}\text { Glaucoma } \\
\text { (original) }\end{array}$ & $\begin{array}{l}\text { Glaucoma } \\
\text { (standardised) }\end{array}$ & $p$ Value (I)* $p$ Value (II)t \\
\hline $\begin{array}{l}\text { Retardation values: } \\
\quad \begin{array}{l}\text { Superior hemiretina } \\
\quad \text { Inferior hemiretina }\end{array}\end{array}$ & $7.61(7.36$ to 7.86$)$ & $7.51(7.24$ to 7.76$)$ & $6.59(6.39$ to 6.80$)$ & 0.55 & $8 \times 10^{-9}$ \\
$\begin{array}{l}\text { Profile shape values: } \\
\quad \text { Superior hemiretina }\end{array}$ & $3.43(9.13$ to 9.73$)$ & $9.26(8.98$ to 9.53$)$ & $8.15(7.92$ to 8.37$)$ & 0.38 & $3 \times 10^{-10}$ \\
$\quad$ Inferior hemiretina & $546(516$ to 576$)$ & $420(396$ to 444$)$ & $385(354$ to 415$)$ & $2 \times 10^{-9}$ & $9 \times 10^{-9}$ \\
\end{tabular}

Retardation values expressed as a mean value in degrees for the appropriate hemiretina.

Profile shape values expressed in degrees (retardation) and represent the sum of the "absolute differences from the mean retardation value" for the 180 degrees comprising a hemiretina.

$95 \%$ Confidence intervals for retardation values and profile shape values in parentheses.

Statistical analysis by Student's $t$ test.

*Compares the normal data with the "original" glaucoma data.

tCompares the normal data with the "standardised" glaucoma data.

measurement of the retinal nerve fibre layer thickness within the appropriate hemiretinas. This can be calculated both before and after standardisation to the normal database for the glaucomatous eyes. These profiles can also be used for the derivation of the profile shape measurement and this can also be calculated both before and after standardisation to the normal database for the glaucomatous eyes.

STATISTICAL ANALYSIS

In this study, one eye from each subject was chosen randomly and used for the statistical analysis. The Student's $t$ test was used to com-

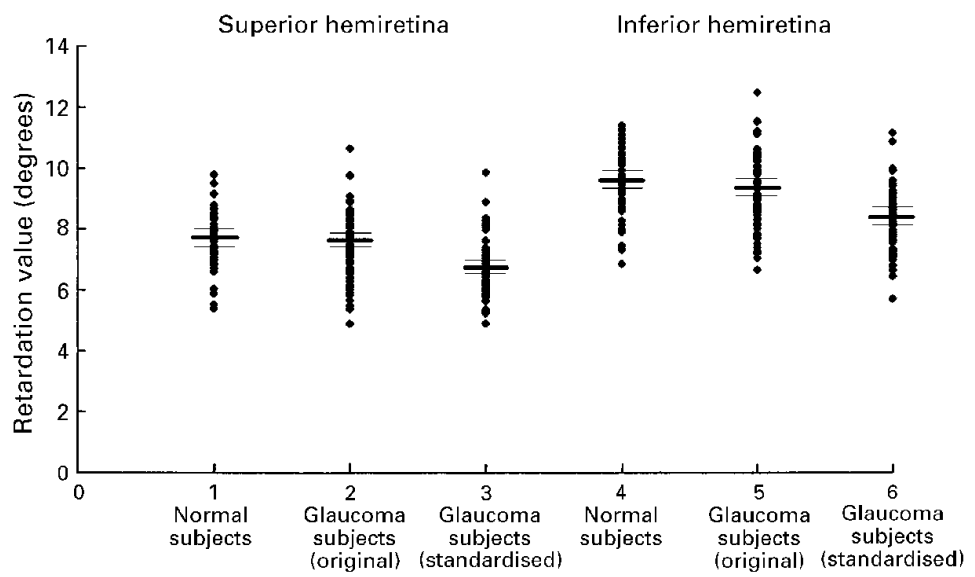

Figure 2 Distribution of the retardation values for the superior and inferior hemiretinas of the normal and glaucomatous eyes. The mean value for each group is demonstrated by the bold line, while the $95 \%$ confidence interval is shown by the fine lines.
Superior hemiretina

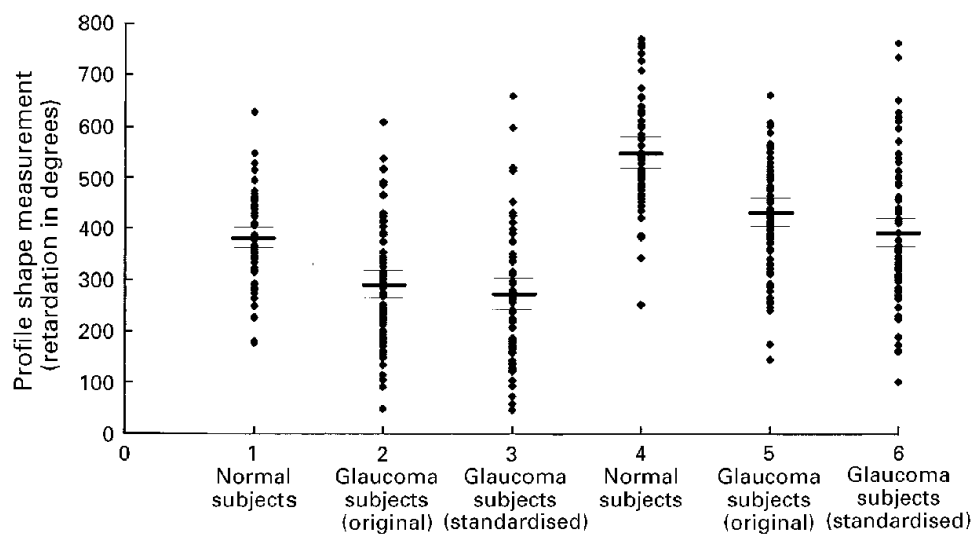

Figure 3 Distribution of the profile shape measurement values for the superior and inferior hemiretinas of the normal and glaucomatous eyes. The mean value for each group is demonstrated by the heavy line, while the $95 \%$ confidence interval is shown by the fine lines. pare the mean age for the two groups. It was also used to compare the results for the normal eyes with those of the glaucomatous eyes both before and after standardisation. The $95 \%$ confidence interval range $(95 \% \mathrm{CI})$ has been calculated and represents the lower and upper 95\% confidence limits for the appropriate data. The sensitivity and specificity values for the mean retardation and profile shape measurements were generated by defining the optimum cut off criterion using the principle of receiver operating characteristic curve analysis. ${ }^{12}$ The Pearson's product moment correlation was used to calculate the correlation of the retardation values and profile shape values to the cup:disc ratios, while Spearman's rank correlation was used to correlate retardation values and profile shape values with visual field mean deviations for the appropriate groups.

\section{Results}

A synopsis of the demographic data of the eyes analysed in the clinical evaluation of this study is given in Table 1. The mean age of the glaucoma group was significantly older at 70 years of age compared with 63 years for the normal group $\left(\mathrm{p}<1 \times 10^{-5}\right)$. The mean cup:disc ratio for the normal subjects was 0.55 compared with 0.75 for the glaucoma group, while the average mean deviation (MD) for the visual field assessment is $0 \mathrm{~dB}$ for the normal subjects and $-8.25 \mathrm{~dB}$ (range of +1.33 to $-28.22 \mathrm{~dB}$ ) for the glaucoma group.

The mean retardation values for the superior and inferior hemiretinas of the normal and glaucomatous groups are demonstrated in Table 2. The inferior retardation values were found to be higher than the superior retardation values in both the normal and glaucomatous eyes. The "original" or "nonstandardised" glaucomatous group of eyes had a superior mean retardation value which was only 0.10 degrees lower than the normal group and an inferior mean retardation value of only 0.17 degrees lower than the normal group. These represent only a $1.2 \%$ and $1.8 \%$ reduction in values from the appropriate normal values respectively and were not found to be significantly different using the Student's $t$ test. However, the standardised glaucomatous group of eyes had a superior mean retardation value which was 1.02 degrees lower than the normal group and an inferior mean retardation value of 1.28 degrees lower than the normal group. These represent a statistically 
Table 3 Standardised retardation values and profile shape values for varying degrees of severity of glaucoma

\begin{tabular}{llll}
\hline & Mild (less than 0.75) & $\begin{array}{l}\text { Moderate }(0.75 \text { to } \\
\text { less than 0.85) }\end{array}$ & Severe (0.85 or greater) \\
\hline Retardation values: & & & \\
$\quad$ Superior hemiretina & 6.94 & 6.55 & 6.29 \\
$\quad$ Inferior hemiretina & 8.41 & 8.38 & 7.70 \\
$\begin{array}{l}\text { Profile shape values: } \\
\quad \text { Superior hemiretina }\end{array}$ & 304 & 266 & 197 \\
$\quad$ Inferior hemiretina & 404 & 379 & 325 \\
\hline Visual field (mean & & Moderate $(-3.5$ to & Severe (-10 dB or worse) \\
$\quad$ deviation) & Mild (up to $-3.5 \mathrm{~dB})$ & $-9.99 \mathrm{~dB})$ & \\
\hline Retardation values: & & & \\
$\quad$ Superior hemiretina & 6.72 & 6.76 & 6.30 \\
$\quad$ Inferior hemiretina & 8.71 & 8.13 & 7.56 \\
Profile shape values: & 279 & 271 & 209 \\
$\quad$ Superior hemiretina & 279 & 392 & 321 \\
$\quad$ Inferior hemiretina & 436 & & \\
\hline
\end{tabular}

Table 4 Optimum sensitivity and specificity values for the retardation values and profile shape values for both hemiretinas

\begin{tabular}{llllll}
\hline & \multicolumn{2}{l}{ Superior hemiretina } & & \multicolumn{2}{l}{ Inferior hemiretina } \\
\cline { 2 - 3 } & Sensitivity & Specificity & & Sensitivity & Specificity \\
\hline $\begin{array}{l}\text { Retardation values: } \\
\quad \begin{array}{l}\text { Normal compared with } \\
\text { original glaucoma }\end{array}\end{array}$ & 54 & & & \\
$\begin{array}{l}\text { Normal compared with } \\
\text { standardised glaucoma }\end{array}$ & 74 & 78 & & 49 & 61 \\
$\begin{array}{l}\text { Profile shape values: } \\
\quad \text { Normal compared with } \\
\text { original glaucoma }\end{array}$ & 72 & 76 & & 73 & 80 \\
$\begin{array}{l}\text { Normal compared with } \\
\text { standardised glaucoma }\end{array}$ & 78 & 76 & & 64 & 81 \\
\hline
\end{tabular}

All sensitivities and specificities given as percentages.

All values optimised with the use of receiver operating characteristic curve analysis.

significant $13.4 \%$ and $13.6 \%$ reduction in values from the appropriate normal values respectively.

The measurements for the profile shape values (measured in degrees) are also documented in Table 2. The inferior values were also found to be higher than the superior values in both the normal and glaucomatous eyes. The original or non-standardised glaucomatous group of eyes had a superior hemiretinal shape value which was 94 degrees lower than the normal group and an inferior hemiretinal shape value which was 126 degrees lower than the normal group. These represent a statistically significant $25 \%$ and $23 \%$ reduction in values from the appropriate normal values respectively. The application of the profile shape algorithm to the standardised glaucomatous polar profiles produced a superior hemiretinal shape value which was 121 degrees

Table 5 Correlation of retardation values and profile shape values to cup:disc ratio and visual field mean deviation

\begin{tabular}{lll}
\hline & Cup:disc ratio & Mean deviation (visual field) \\
\hline Retardation values: & & \\
$\quad$ Normal & $+0.07 ; \mathrm{p}>0.1(0.5 \%)$ & $-0.03 ; \mathrm{p}>0.1$ \\
Glaucoma (original) & $-0.08 ; \mathrm{p}>0.1(0.6 \%)$ & $+0.15 ; \mathrm{p}>0.1$ \\
Glaucoma (standardised) & $-0.40 ; \mathrm{p}<0.01(16 \%)$ & $+0.44 ; \mathrm{p}<0.01$ \\
Profile shape values: & $+0.01 ; \mathrm{p}>0.1(0.0 \%)$ & $-0.05 ; \mathrm{p}>0.1$ \\
$\quad$ Normal & $-0.38 ; \mathrm{p}<0.01(14 \%)$ & $+0.39 ; \mathrm{p}<0.01$ \\
Glaucoma (original) & $-0.35 ; \mathrm{p}<0.01(13 \%)$ & $+0.35 ; \mathrm{p}<0.01$ \\
Glaucoma (standardised) &
\end{tabular}

Statistical analysis used for the above correlations:

Correlation with cup:disc ratio = Pearson's product moment correlation with Student's $t$ test for statistical significance and the coefficient of determination $\left(r^{2}\right)$ expressed in percentages and in parentheses.

Correlation with visual field $=$ Spearman's rank correlation coefficient with statistical significance . lower than the normal group and an inferior hemiretinal shape value which was 161 degrees lower than the normal group. These also represent a statistically significant $32 \%$ and $29 \%$ reduction in values from the appropriate normal values respectively.

The distribution of the retardation values obtained for the normal and glaucomatous eyes in both the superior and inferior hemiretinas are illustrated in Figure 2, while the distribution of the profile shape values obtained for the same groups are illustrated in Figure 3. The $95 \%$ confidence intervals for the various groups are also displayed. Table 3 illustrates the standardised mean retardation and profile shape values following the stratification of the glaucoma patients into three categories. The severity of the glaucoma has been graded both in terms of the cup:disc ratio and the visual field mean deviation, while each category has at least 20 eyes comprising the mean values. In general, there is an appropriate reduction in both the retardation and the profile shape values with an increasing severity of glaucoma, the pattern being more apparent with an anatomical stratification.

Table 4 documents the sensitivity and specificity values for the mean retardation and profile shape measurements for the appropriate hemiretinas. These pairs of percentages represent the optimum diagnostic performance for the individual groups, based upon using the receiver operating characteristic curve analysis to identify the best cut off criterion in each case. There is a general improvement in the performance of both the retardation values and the profile shape values following the standardisation procedure. The inferior hemiretina provided slightly better outcomes, with the best sensitivity and specificity being $70 \%$ and $91 \%$ respectively for the standardised profile shape measurement of the inferior hemiretina. The cut off criterion for this pair of percentages was 430 degrees (of profile shape).

The correlation coefficients corresponding to the correlation of retardation values and profile shape measurement values (for the whole eye rather than individual hemiretinas) to the cup:disc ratio and visual field mean deviations for the normal and glaucomatous eyes are demonstrated in Table 5. The correlation of the normal subjects to the two traditional variables is very weak, both in terms of the retardation values and in the profile shape. Equally, the non-standardised glaucoma retardation values are also very weakly correlated with both cup:disc ratio and visual field mean deviations. However, the nonstandardised glaucoma shape measurements are significantly correlated with the cup:disc ratio at $r=-0.38(\mathrm{p}<0.01)$ and the visual field $\mathrm{MD}$ at $r=+0.39(\mathrm{p}<0.01)$. The standardisation of the glaucomatous retardation values has a significant effect on the correlation of these values with the other variables $(r=-0.40, \mathrm{p}$ $<0.01$ for cup:disc ratio; $r=+0.44, \mathrm{p}<0.01$ for $\mathrm{MD})$. However, standardisation has no enhancing effect on the correlation of the glaucomatous shape measurements with these variables. 


\section{Discussion}

The initial research on the clinical evaluation of the scanning laser polarimeter ${ }^{9} 10$ has confirmed previous reports ${ }^{4}$ that the retardation images of normal eyes demonstrate some global features consistent with known features of the retinal nerve fibre layer. Furthermore, it has also been shown that the interpretation of the retardation values obtained from subjects can be significantly improved by the application of a blood vessel removal algorithm, ${ }^{78}$ a feature which the manufacturers have now incorporated into their Mark II version of this instrument. ${ }^{13}$ The authors have also demonstrated that more sophisticated analyses of the raw retardation values, especially with regard to the shape of the polar profiles, can lead to a better discrimination of normal and glaucomatous eyes. ${ }^{910}$

This study was performed in an attempt to improve on the previous methods devised by the authors to assess the retardation values comprising polar profiles and the shape of these polar profiles. Two relatively simple algorithms have been devised to achieve this evaluation. The first algorithm standardises an individual's polar profile to a known eye matched normal database profile, while the second algorithm uses a simple mathematical calculation to provide a measure of the polar profile shape.

An assessment of the raw retardation values obtained from a sample of normal and glaucomatous subjects confirms previous findings ${ }^{9} 10$ that there is less than a $2 \%$ reduction in the hemiretinal retardation values in the glaucoma group compared with the normal group. The authors had previously used the nasal quadrant as a reference point from which to standardise the retardation values and this had improved the discrimination between these two groups ${ }^{9}{ }^{10}$ In this study, standardisation to the nasal and temporal 30 degrees also improves the discrimination of these two groups to a high degree of significance, with a $13.5 \%$ difference in the hemiretinal retardation values. The sensitivity and specificity of these standardised values are substantially improved over the original retardation measurements. Furthermore, the mean values demonstrate a gradual decline with increasing severity of glaucoma.

The standardisation procedure is one method of overcoming the previously reported observations $^{910}$ that the NFA I measures greater retardation values in the temporal and nasal regions of glaucoma subjects compared with normal subjects. However, this method relies on the availability of a normal database, which ideally is age and eye matched. In this study, we utilised our own small sample of normal subjects as there was no commercially available normal database at the time. Furthermore, it requires the definition of a reference point or range, a feature which may encounter problems similar to those observed by users of the scanning laser ophthalmoscope.

An alternative approach is to assess the shape of the polar profile. This is made possible by the application of a blood vessel removal algorithm, a method which the au- thors have previously described ${ }^{7}$ and a process which the manufacturers have now incorporated into the scanning laser polarimeter. ${ }^{13}$ Previous research ${ }^{9}{ }^{10}$ has highlighted the potential that polar profile shape analysis offers in the discrimination of normal and glaucomatous subjects.

In this study, a simple algorithm was designed to provide a more comprehensive assessment of the shape or modulation of the polar profile. The results demonstrate that by using this simple algorithm on the nonstandardised retardation values, the discrimination between the normal and glaucoma subjects is improved, with a statistically significant $24 \%$ difference between the two groups. This difference can be increased by the use of the standardised retardation values to the order of $30 \%$. Furthermore, the profile shape measurements also demonstrate a gradual decline in value with increasing severity of glaucoma.

Despite these improvements, there is still no one variable which demonstrates mutual exclusivity between these two groups (see Figs 2 and 3). This is illustrated in a more diagnostic manner by the optimal sensitivities and specificities for this study. All of the pairs of percentages are relatively poor with regard to the diagnostic use of any single variable. Even the best outcome $(70 \%$ sensitivity, $91 \%$ specificity) is still inferior to that previously documented for the more traditional method of the glaucoma hemifield test $(94 \%$ sensitivity, $90 \%$ specificity $^{14}$ ), but is slightly better than red-free photography (64\% sensitivity, $84 \%$ specificity $\left.^{15}\right)$. Interestingly, the ranges of sensitivities and specificities obtained in this study are also substantially lower than the $96 \%$ sensitivity and $93 \%$ specificity recently reported for the diagnostic performance of the NFA I. ${ }^{16}$ The reasons for such a discrepancy are unclear, but may reflect the differing methods of data retrieval from the retardation images, the individual study's definition of abnormality, and the actual derivations of the sensitivity and specificity values.

The authors also considered it important to compare the original and modified retardation values and profile shape measurements with the traditional methods of assessing glaucoma subjects - namely, the cup:disc ratio and the visual field. In general, the non-standardised retardation values were extremely weakly correlated with both of the traditional variables. The standardisation of the glaucoma retardation values improves the correlation to a significant but modest $r=-0.4(\mathrm{p}<0.01)$ with the cup:disc ratio and $r=+0.44(\mathrm{p}<0.01)$ with the mean deviation of the visual fields. Similar correlation coefficients are obtained with the profile shape measurement using nonstandardised retardation values.

These results are comparable with those of other studies ${ }^{56}$ using different methods to utilise the raw retardation values. Nevertheless, these results are still rather disappointing, in that only approximately $15 \%$ of the variation in the retardation values is attributable to the cup:disc ratio or the mean deviation of the visual field. This may be explained by the 
relatively subjective and variable assessment of the cup:disc ratio, or by the chronological delay in functional loss in patients with glaucoma. However, it may also be a reflection on the retardation values themselves. We have previously alluded to the question of the exact relation between the retardation values which probably represent microtubule density in the retinal nerve fibre layer and the retinal nerve fibre layer thickness itself. ${ }^{10}$ Furthermore, we have demonstrated both clinically ${ }^{910}$ and in a primate study $^{17}$ that there is a disparity between the exact location of the retinal nerve fibre layer and the characteristic "double hump" pattern of the polar profiles.

The scanning laser polarimeter is a new method for quantifying retinal nerve fibre layer thickness. The interpretation of the retardation values obtained with this instrument can be improved significantly by either standardising an individual's profile to a normal database or by assessing the polar profile shape. Both of these methods will now be possible with the GDx, the latest version of the scanning laser polarimeter, following the recent development by the manufacturers of their own blood vessel removal algorithm ${ }^{13}$ and the construction of a normal database. However, the modest sensitivity and specificity values obtained in this study, along with the relatively weak correlation of these new methods of interpreting the polar profiles with conventional variables, highlights the need to determine exactly what the scanning laser polarimeter actually measures. The authors would like to acknowledge the work of Mrs Gill
Bennerson who performed the optic disc photographic Bennerson who performed the optic disc photographic
documentation of these patients, along with her advice and supdocumentation of these patients, along with her advice and sup-
port throughout this project. This study was supported by the port throughout this project. This study was supported by the
Special Trustees for the United Bristol Healthcare Trust and Special Trustees for the
the UK Pacesetter Fund.

The authors have no financial or proprietary interest in the
The Pacesetter Fund. scanning laser polarimeter (Nerve Fiber Analyzer (NFA I)) or Laser Diagnostic Technologies Inc, San Diego, California, USA.
1 Weinreb RN, Dreher AW, Coleman, A, et al. Histopathologic validation of Fourier-ellipsometry measurements of retinal nerve fibre layer thickness. Arch Ophthalmol 1990;108:557-60.

2 Dreher AW, Reiter K. Retinal laser ellipsometry: a new method for measuring the retinal nerve fibre layer thickness distribution. Clin Vis Sci 1992;7:481-8.

3 Dreher AW, Reiter K, Weinreb RN. Spatially resolved birefringence of the retinal nerve fibre layer assessed with retinal laser ellipsometer. Appl Optics 1992;31:3730-5.

4 Weinreb RN, Shakiba S, Zangwill L. Scanning laser polarimetry to measure the nerve fibre layer of normal and glaucomatous eyes. Am f Ophthalmol 1995;119:627-36.

5 Weinreb RN, Shakiba S, Sample PA, et al. Association between quantitative nerve fibre layer measurement and visual loss in glaucoma. Am f Ophthalmol 1995;120:732-8.

6 Niessen AGJE, van der Berg TJTP, Langerhorst CT, Greve EL. Retinal nerve fibre layer assessment by scanning laser polarimetry and standardized photography. Am f Ophthalmol 1996;121:484-93.

7 Waldock A, Karwatowski WSS, Potts MJ. Improving the reproducibility of the nerve fiber analyzer retinal nerve fibre layer profiles with a blood vessel removal algorithm. Invest Ophthalmol Vis Sci (Suppl) 1995;36:S973.

8 Waldock A, Potts MJ, Sparrow JM, Karwatowski WSS. Clinical evaluation of scanning laser polarimetry:I. Intraoperator variability and design of a blood vessel removal algorithm. (submitted for publication)

9 Waldock A, Karwatowski WSS, Potts MJ. Clinical interpretation of the nerve fibre layer profiles obtained with the scanning laser polarimeter. Invest Ophthalmol Vis Sci (Suppl) 1996;37:S1095.

10 Waldock A, Potts MJ, Sparrow JM, Karwatowski WSS. Clinical evaluation of scanning laser polarimetry: II Interpretation of retardation values in a group of normal and glaucoma patients. (submitted for publication)

11 Dreher AW, Reiter K. Scanning laser polarimetry of the retinal nerve fibre layer. Proc SPIE Int Soc Opt Eng 1992;1746: 34-8.

12 Zweig MH, Campbell G. Receiver-operating characteristic (ROC) plots:a fundamental tool in clinical medicine. Clin Chem 1993;39:561-77.

13 Dreher, AW, Reed GC, Zyzyck J, Zhou Q, Reiter K. Reproducibility improvement of the nerve fiber analyzer by applying a blood vessel removal algorithm. Invest Ophthalmol Vis Sci (Suppl) 1996;37:S1093.

14 Katz J, Sommer A, Gaasterland DE, Anderson DR. Comparison of analytic algorithms for detecting glaucomatous visual field loss. Arch Ophthalmol 1991;109:1684-9.

15 Wang F, Quigley HA, Tielsch JM. Screening for glaucoma in a medical clinic with photographs of the nerve fibre layer. Arch Ophthalmol 1994;112:796-800.

16 Tjon-Fo-Sang MJ, Lemij HG. The sensitivity and specificity of nerve fibre layer measurements in glaucoma as determined with scanning laser polarimetry. Am f Ophthalmol 1997;123:62-9.

17 Morgan JE, Waldock A, Jeffrey C, Cowey A. Retinal nerve fibre layer polarimetry: a histological and clinical comparion. (submitted for publication) 